Néia Schor 1

Andrea Felicissimo Ferreira 1

Vera L. Machado 1

Ana Paula França 1

Kátia C. M. Pirotta 1

Augusta Thereza de Alvarenga 1

Arnaldo Augusto Franco de Siqueira 1

\section{Mulher e anticoncepção: conhecimento e uso de métodos anticoncepcionais}

\author{
Women and contraception: \\ knowledge and use of contraceptive methods
}

1 Departamento de Saúde Materno-Infantil,

Faculdade de Saúde Pública, Universidade de São Paulo. Av. Dr. Arnaldo 715, 2o andar, sala 218, São Paulo, SP 01246-904, Brasil. afelici@yahoo.com.br

\begin{abstract}
This article analyzes knowledge and use of contraceptive methods in women ages 10 to 49 years residing in the southern region of the city of São Paulo in 1992. A total of 1,157 childbearing-age women were studied, focusing on variables that might define them as to: knowledge in the use of contraceptive methods and reasons for choosing a given method. We observed that 86\% of the women referred knowledge of some contraceptive method, while the most common was the pill (95.3\%), followed by condoms (92.6\%). Meanwhile, 14\% of the interviewees denied knowledge of any contraceptive method. Of the sexually active women (66.4\%), 34.9\% reported never having used contraceptive methods. Of those who had, 35.3\% used the pill, while $42.9 \%$ had resorted to sterilization. Only 5.2\% used condoms. Despite the high level of knowledge concerning contraceptive methods, especially oral contraceptives and condoms, we observed limited use of same as compared to the high sterilization rate around the age of 27 , thus leaving contraception limited to the pill and female sterilization.
\end{abstract}

Key words Contraceptive Devices; Contraception; Sex Behavior

Resumo Neste estudo buscou-se fazer uma análise do conhecimento e do uso de métodos anticoncepcionais (MAC) entre mulheres de 10 a 49 anos residentes na Região Sul do Município de São Paulo, no ano de 1992. Foram estudadas 1.157 mulheres em idade reprodutiva, tendo em vista variáveis que pudessem caracterizá-las quanto ao conhecimento de métodos anticoncepcionais (MAC), ao seu uso e ao motivo pelo qual optaram por determinado método. Verificou-se que $86 \%$ das mulheres desse universo referiram conhecer algum MAC, sendo a pílula o mais citado (95,3\%) e, em segundo lugar, o condom masculino, que obteve 92,6\% das respostas. Dentre as mulheres, $14 \%$ relataram não conhecer nenhum MAC. Das mulheres pesquisadas, 66, $4 \%$ têm vida sexual e, dessas, 65,1\% fazem uso de algum tipo de MAC. A pílula é usada por 35,3\% das usuárias de MAC, enquanto que 42,9\% estão esterilizadas. Quanto ao uso do condom masculino, apenas 5,2\% referem o uso. Apesar do alto índice de conhecimento de MAC-sobretudo, a pílula e o condom masculino - constatou-se baixo uso dos mesmos associado à alta freqüência de mulheres esterilizadas, ficando a anticoncepção circunscrita à pílula e à esterilização feminina.

Palavras-chave Dispositivos Anticoncepcionais; Anticoncepção; Comportamento Sexual 


\section{Introdução}

A realidade da anticoncepção para a mulher brasileira tem suscitado discussões diversas nos últimos anos, as quais envolvem desde aspectos sociais - pois as mulheres estão inseridas em um quadro de desigualdade de direitos, de oportunidades e de recursos financeiros até aspectos políticos, uma vez que os programas de atenção a sua saúde não estão efetivamente implementados (Berquó et al., 1985; Morell, 1994; Schor, 1995). O resultado é o agravamento do quadro de saúde no Brasil quanto à realidade das práticas contraceptivas.

Os programas de atenção à saúde da mulher no país voltavam-se tradicionalmente para o grupo materno-infantil, enfocando as condições de gestação e do parto para garantir uma prole sadia. Por sua vez, o Programa de Assistência Integral à Saúde da Mulher - PAISM surgiu em 1983 - com base no conceito de Atenção Integral à Saúde da Mulher - AISM -, como proposta inovadora que rompia com a visão tradicional, passando a privilegiar a mulher no atendimento das questões ligadas à reprodução (Osis, 1994).

O PAISM, que fora regulamentado em 1986, resultou de esforços dos profissionais da área de saúde no sentido de preconizar ações que ampliassem o atendimento à saúde das mulheres. Sob essa óptica, a atenção à mulher deveria ser integral, podendo contar com exames para detectar câncer cérvico-uterino e de mama, controle de DST, assim como assistência para o planejamento familiar, que inclui tanto a infertilidade como a contracepção.

No entanto, o programa tem sofrido críticas de diversos autores em função dos problemas de implementação de suas diretrizes. É bem verdade que não há como deixar de reconhecer a importância do PAISM na abordagem à saúde reprodutiva no Brasil, mas é sabido que a realização efetiva do programa em todo o território nacional não aconteceu. A análise dos fatos indica a ocorrência de descompasso entre as bases programáticas do PAISM e as ações práticas, apontando para o descomprometimento político com a efetiva execução desse programa (Osis, 1994).

Nesse sentido, as mulheres que dependem do sistema público de saúde dispõem de poucos recursos para regular sua fecundidade e, apesar da disponibilidade de vários métodos contraceptivos, ficam restritas ao uso da pílula e à esterilização feminina, como apontam vários estudos (Berquó, 1993; Morell, 1994; Schor \& Morell, 1994; Schor, 1995; Pirotta, 1998), muito embora a esterilização não deva ser consi- derada método anticoncepcional, por ser praticamente irreversível. Portanto, a falta de comprometimento das políticas de saúde com as necessidades da população geram o agravamento do quadro de saúde no país quanto à realidade das práticas contraceptivas.

A falta de expectativas está levando a população a distanciar-se cada vez mais tanto de seus direitos, quanto do acesso à qualidade mínima de vida, ao menos no que diz respeito à saúde e à educação.

\section{Objetivo}

Este trabalho tem por objetivo caracterizar o conhecimento e o uso de métodos anticoncepcionais (MAC) entre as mulheres (10 a 49 anos) residentes na Região Sul do Município de São Paulo, Brasil, em 1992, segundo variáveis de natureza biológica, demográfica e social.

\section{Metodologia}

O presente estudo está inserido em projeto maior, intitulado "Morbidade e Mortalidade Maternas, Qualidade de Assistência e Estrutura Social: Estudo da Região Sul do Município de São Paulo. Parte II - Morbidade" (Siqueira et al., 1993; Schor, 1995), cuja investigação subdividiu-se em duas partes: iniciou com o estudo de todos os óbitos ocorridos em 1989 relativos a mulheres de 10 a 49 anos residentes na região sul do município; em seguida, passou a estudar a morbidade de mulheres na mesma faixa etária e residentes na região no ano de 1992, enfatizando a morbidade materna e a saúde reprodutiva.

A Região Sul abarca os subdistritos de Santo Amaro e Capela do Socorro e o distrito de Parelheiros; é a mais extensa de São Paulo, correspondendo a $633,7 \mathrm{~km}^{2}$, o que eqüivale a $42 \%$ da área do município. Segundo o Censo de 1991, a sua população era de aproximadamente 1.662.231 habitantes, distribuídos de forma heterogênea (IBGE, 1991).

Para as finalidades da segunda parte do projeto - Morbidade - procedeu-se a uma subdivisão por áreas, considerando-se a taxa de mortalidade infantil e feminina (Unglert et al., 1990; Siqueira et al., 1991). Assim, a partir da subdivisão do território proposta por Unglert (1986) - com base na fusão de setores censitários mediante critérios geográficos, demográficos e sociais, presença de barreiras naturais ou construídas etc. -, a região foi desmembrada em 31 áreas com características comuns. 
A Região Sul do Município de São Paulo (SMSP) caracteriza-se, como já descrito, pela vasta extensão territorial e por possuir grande contingente populacional. Assim, o estudo da população feminina de 15 a 49 anos nela residente exigiu que a amostra não fosse apenas representativa da totalidade, mas que possibilitasse preservar as diversidades entre as áreas.

Com esse propósito foram adotados, como parâmetros, o estudo de mortalidade de mulheres de 10 a 49 anos efetuado por Siqueira et al. (1991) e o de mortalidade infantil realizado por Unglert et al. (1990), ambos concernentes à população residente na região. Ao serem cruzados esses dois coeficientes, foram obtidos oito tipos de agrupamentos de áreas que se referiam a características combinadas de alta, média e baixa mortalidade feminina/infantil. A partir dessa nova composição das áreas, procedeu-se ao sorteio de cada tipo de agrupamento de modo a garantir a presença das áreas com diferentes níveis de mortalidade. A amostra teve onze áreas sorteadas, as quais foram subdivididas em sessenta quarteirões. Realizou-se então o reconhecimento de cada um destes com o objetivo de verificar se a área era residencial ou comercial.

A unidade amostral por área sorteada foi a residência de mulheres em idade fértil, isto é, de 10 a 49 anos. A amostra foi determinada pelo número de habitantes, aplicando-se um questionário para cada dois mil habitantes. No subgrupo em que a população era menor que 10.000 habitantes, definiu-se que seriam aplicados questionários em, no mínimo, cinco casas com mulheres de 10 a 49 anos.

A amostra da pesquisa compreendeu 727 domicílios onde residiam 750 famílias, totalizando 3.268 indivíduos. Destes, 1.733 eram do sexo feminino, sendo que 1.261 estavam na faixa etária dos 10 aos 49 anos. O número de entrevistas completas, contendo dados relativos à vida reprodutiva das mulheres, totaliza 1.157 entrevistas (Figura 1), porque 104 mulheres não foram encontradas no local de residência após três visitas. A idade das mulheres foi agrupada em faixas etárias de cinco anos para facilitar a análise dos dados.

Por motivos éticos, a equipe de pesquisa estabeleceu que as adolescentes com idade inferior a 15 anos não seriam indagadas a respeito de temas que abordassem a vida sexual, a menos que estivessem grávidas ou que já tivessem filhos; caso contrário, estas responderiam apenas as questões acerca do conhecimento de métodos anticoncepcionais.

O grupo privilegiado neste estudo abrange 1.157 mulheres de 10 a 49 anos. Trabalhou-se com dados relativos ao conhecimento de MAC, que foram levantados a partir de dois procedimentos: inicialmente perguntou-se quais métodos contraceptivos a mulher conhecia e, em seguida, o entrevistador enumerou aqueles que não tivessem sido citados espontaneamente pela mulher, para que ela confirmasse se conhecia ou não. O primeiro tipo de resposta foi designado como "resposta espontânea" e o segundo, "resposta estimulada". Em relação ao tipo de MAC foram tomados em conta os métodos citados pelas mulheres independente de sua eficácia, pois não era propósito da pesquisa investigar a eficiência do MAC citado. Em um segundo momento trabalhou-se com todas as mulheres que já haviam iniciado vida sexual, ou seja, aquelas que referiram ter tido pelo menos uma relação sexual - totalizando 769 mulheres na faixa etária de 15 a 49 anos - em relação à variável uso de MAC, tipo de MAC citado no momento da entrevista, o motivo da escolha e a indicação de uso.

\section{Resultados e discussão}

\section{Conhecimento de Métodos \\ Anticoncepcionais (MAC)}

Diversos autores apontam para o fato de que o início da atividade sexual tem ocorrido na adolescência. Em estudo concernente à anticoncepção e adolescência com a mesma população, Schor (1995) observou que, quanto menor a idade da adolescente ao iniciar a vida sexual, menor a chance de ela estar usando algum MAC e, conseqüentemente, maior a probabilidade de ficar grávida logo nas primeiras relações.

A autora também advertiu que há alto nível de conhecimento de MAC entre a população de adolescentes, embora não tenha sido avaliada a qualidade do conhecimento. A falta de planejamento e a esporadicidade com que ocorrem as relações sexuais são aspectos que influenciam o não uso de MAC. Para a população como um todo essa realidade não difere muito; as mulheres relatam conhecer diferentes métodos contraceptivos, mas o uso fica restrito a apenas dois deles, como será visto no decorrer do trabalho.

Dentre as mulheres entrevistadas, $86,4 \%$ narraram conhecer MAC e 13,6\%, nunca ter ouvido falar em métodos contraceptivos. Vale ressaltar que, entre as jovens de 10 a 14 anos, há baixa percentagem de conhecimento (48,3\%), o que pode ser explicado pelo fato de a jovem ainda não ter iniciado vida sexual, não mani- 


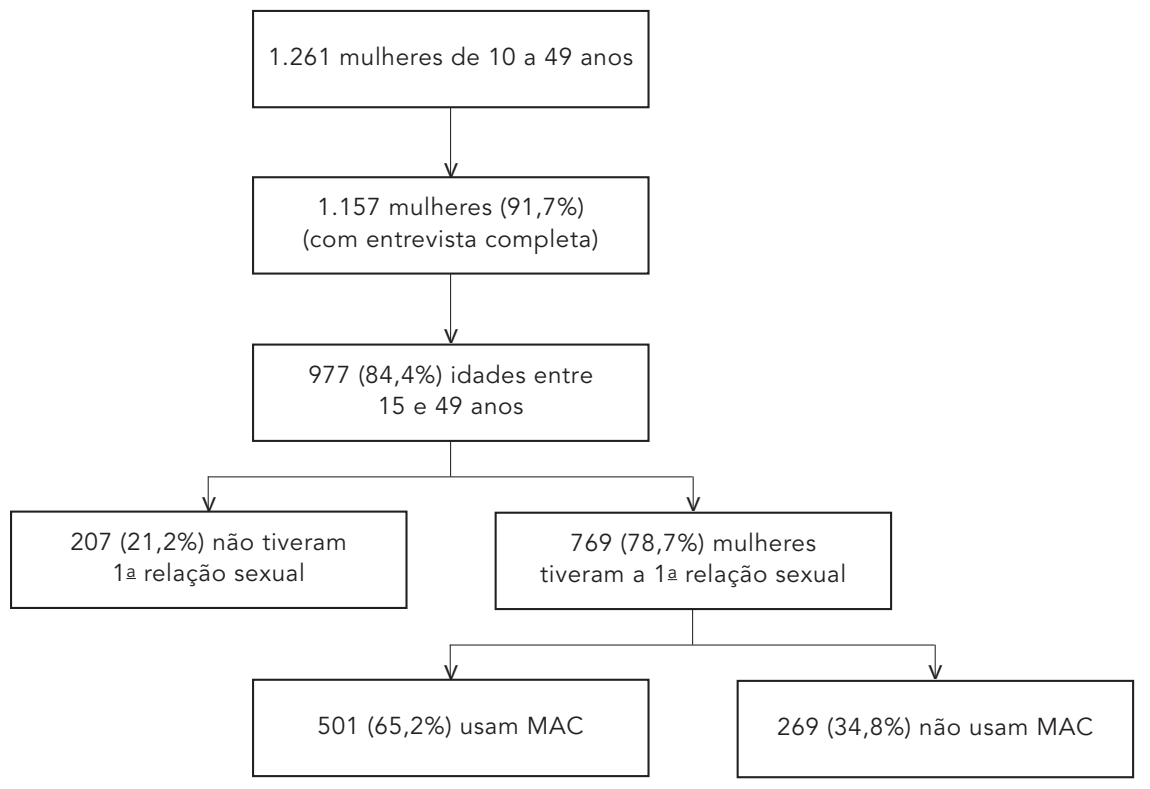

festando preocupação com MAC. Em contrapartida ocorre um salto a partir dos 15 anos, quando $92,2 \%$ das jovens expuseram que conheciam maneiras de evitar gravidez. A Tabela 1 ilustra quais são os métodos contraceptivos mais “conhecidos/referidos", por faixa etária, pelas mulheres no momento da entrevista.

Segundo dados da Pesquisa Nacional sobre Demografia e Saúde - PNDS, de 1996: “... praticamente $100 \%$ dos entrevistados, homens e mulheres - sejam estes unidos ou não, e com ou sem experiência sexual-conhecem, de certa forma, algum tipo de método" (BEMFAM, 1997:48).

Na Região Sul do Município de São Paulo, o preservativo/condom masculino e a pílula são citados por todas as mulheres entrevistadas em proporção de 95,3\% e 92,6\%, respectivamente. Em seguida, a esterilização é citada por $78,4 \%$; a tabelinha, por $70,1 \%$; a vasectomia, por $66,6 \%$; e, em último lugar, o DIU, cujo conhecimento é de $64,8 \%$. Constatou-se que os MAC mais conhecidos são o preservativo/condom masculino e a pílula. O conhecimento a respeito de ambos entre as adolescentes com idade menor ou igual a 15 anos é superior a $96 \%$. Tal fato pode ser explicado se for considerado que mais de dois terços das jovens relataram ter "ouvido falar" de camisinha e pílula na escola, enquanto as mais velhas, que já se en- contram fora do sistema educacional, deram o círculo feminino como referência principal (Schor, 1995). A partir dos 20 anos, a esterilização obteve conhecimento superior a 85,0\%, mas, na faixa etária de 15 a 19 anos, 73,3\% das mulheres a conheciam. Quando analisada a vasectomia - esterilização masculina - pode-se perceber que o conhecimento das mulheres no que lhe diz respeito é menor $(66,6 \%)$; somente a partir dos 25 anos é que se torna superior a $80,0 \%$.

A abstinência periódica (tabelinha) é conhecida por $70,1 \%$ das mulheres. Cerca de $17,9 \%$ das mulheres na faixa etária de 10 a 14 anos a conhecem. Na faixa etária seguinte, tal percentagem sobe para $62,8 \%$ e, a partir dos 20 anos, é superior a 80,0\%. Em geral, a informação quanto a esse tipo de MAC tem sido ainda fornecida, em grande parte, em âmbito popular, estando os serviços de saúde ao largo desse processo, ou, melhor dizendo, enclausurados em si mesmos ou em suas rotinas administrativas, talvez indiferentes às necessidades relacionadas à anticoncepção (Schor, 1995), em particular, na adolescência, que constitui período importante para a transmissão de informação. 
Distribuição percentual do conhecimento dos MAC mais referidos, segundo faixa etária.

\begin{tabular}{|c|c|c|c|c|c|c|}
\hline Faixa etária & Condom & Pílula & Esterilização & Vasectomia & DIU & $\begin{array}{l}\text { Abstinência } \\
\text { periódica }\end{array}$ \\
\hline $10-14$ & 81,2 & 66,7 & 35,7 & 20,3 & 11,6 & 17,9 \\
\hline $15-19$ & 97,8 & 96,1 & 73,3 & 63,3 & 49,5 & 62,8 \\
\hline $20-24$ & 98,8 & 98,2 & 86,5 & 74,2 & 71,8 & 81,6 \\
\hline $25-29$ & 98,1 & 99,4 & 91,6 & 81,3 & 82,9 & 88,4 \\
\hline $30-34$ & 97,8 & 98,5 & 91,1 & 82,1 & 89,6 & 88,1 \\
\hline $35-39$ & 98,4 & 99,2 & 93,0 & 82,2 & 88,4 & 86,1 \\
\hline $40-44$ & 98,9 & 100,0 & 94,7 & 83,0 & 88,3 & 87,2 \\
\hline $45-49$ & 99,0 & 96,9 & 91,6 & 77,9 & 74,7 & 84,2 \\
\hline $10-49$ & 95,3 & 92,6 & 78,4 & 66,6 & 64,8 & 70,1 \\
\hline
\end{tabular}

\section{Uso de Métodos Anticoncepcionais}

Dentre as mulheres entrevistadas, $78,8 \%$ narraram ter vida sexual. Atentando-se apenas para aquelas com vida sexual, $65,2 \%$ referiram o uso de algum tipo de MAC e 34,8\% relataram não fazer uso.

A Figura 2 ilustra os métodos contraceptivos mais utilizados pelas mulheres na época da entrevista. Há grande concentração em termos da esterilização (42,9\%) e da pílula (35,3\%). A presença de outros tipos de MAC dá-se em menor proporção: o preservativo $(5,2 \%)$, a vasectomia $(4,8 \%)$ e o DIU $(1,6 \%)$. Os outros métodos citados correspondem a métodos de baixa eficácia, como a abstinência periódica/tabelinha $(1,4 \%)$ e o coito interrompido $(2,2 \%)$.

Segundo dados da PNDS de 1996, embora se observe alta prevalência de uso de contraceptivos no país, $40,2 \%$ das mulheres brasileiras com idade entre 15 e 49 anos tinham recorrido à esterilização para controlar sua fecundidade. Ao lado destas, $23,2 \%$ não estavam usando MAC, $20,7 \%$ utilizavam a pílula, 6,6\% recorriam a métodos tradicionais, $4,4 \%$ usavam preservativo, $2,6 \%$ a esterilização masculina, 1,2\% a injeção e 1,1\% o DIU (BEMFAM, 1997). Este panorama indica a falta de opções contraceptivas a que as mulheres estão sujeitas.

Na Tabela 2 nota-se que o uso de MAC praticamente está restrito à esterilização (42,9\%) e à pílula $(35,3 \%)$, muito embora a primeira não deva ser considerada método contraceptivo por seu caráter praticamente irreversível. Na faixa etária dos 15 aos 29 anos há grande concentração de usuárias de pílula; já as mulheres esterilizadas, a partir dos 30 anos, superam a percentagem das usuárias de pílula. O uso do preservativo/condom masculino está aquém
Figura 2

Distribuição da porcentagem das mulheres que iniciaram a vida sexual, usuárias de MAC, segundo tipo de MAC utilizado.

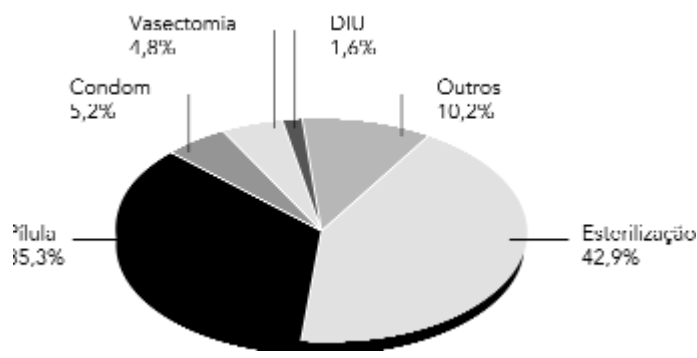

do esperado em todas as faixas etárias, tendo em vista as DSTs e, principalmente, a AIDS. A opção pelo DIU é baixa em todas as faixas etárias, assim como a vasectomia.

Segundo Morell (1994:229): "O tipo de método usado pelas mulheres no Estado de São Paulo, em suma, modifica-se com o avançar da idade. As mais jovens privilegiam o uso de métodos reversíveis. A partir dos 30 anos, optam por métodos definitivos. O uso da pílula decresce à medida que a idade aumenta, ao passo que a esterilização percorre o caminho inverso, isto é, fica cada vez mais prevalente ao se incrementar o grupo etário".

Comentando os resultados de pesquisa voltada para a realidade da mulher esterilizada na RSMSP em comparação com as usuárias de métodos reversíveis, Pirotta (1998:83) afirma: “Os resultados descritos no presente estudo le- 
vam-nos a considerar que a mulher paulista e a mulher residente na Região Sul do Município de São Paulo estão percorrendo um caminho bastante similar em termos de contracepção, no decorrer da sua história de vida. Este percurso está marcado pelo desejo de controlar a fecundidade que não encontra, junto ao sistema de saúde, outras alternativas anticoncepcionais concretas além do uso da pílula e da esterilização".

Existem fatores relevantes para a opção contraceptiva das mulheres deste estudo. $\mathrm{Na}$ Figura 3 encontra-se a distribuição das mulheres segundo o motivo da escolha do MAC que usam atualmente. Observa-se que $28,0 \%$ das mulheres optaram por determinado método por achá-lo mais seguro e $27,8 \%$, por achá-lo mais prático/fácil de usar. Das mulheres, 9,6\% responderam que usam determinado MAC atualmente por não se terem adaptado à pílula. Quanto à atuação dos profissionais de saúde, 8,0\% das mulheres referiram usar MAC em virtude de ter sido indicado por um médico. Em relação aos outros motivos de escolha, $5,0 \%$ relataram não querer engravidar. Entre os demais motivos citados, 2,3\% das mulheres optaram por um método em razão de ser o mais barato disponível no mercado.

Tabela 2

Distribuição percentual das mulheres com vida sexual, usuárias de MAC, segundo faixa etária e tipo de MAC utilizado.

\begin{tabular}{|c|c|c|c|c|c|c|c|}
\hline Faixa etária & Esterilização & Pílula & Preservativo & Vasectomia & DIU & Outros & Total \\
\hline $15-19$ & - & 85,2 & 3,7 & - & 3,7 & 7,4 & 100,0 \\
\hline $20-24$ & 1,4 & 72,9 & 12,9 & - & 1,4 & 11,4 & 100,0 \\
\hline $25-29$ & 23,7 & 48,4 & 4,3 & 6,5 & - & 17,2 & 100,0 \\
\hline $30-34$ & 42,7 & 32,3 & 2,1 & 8,3 & 5,2 & 9,4 & 100,0 \\
\hline $35-39$ & 64,1 & 16,3 & 5,4 & 4,3 & - & 9,8 & 100,0 \\
\hline $40-44$ & 72,7 & 10,6 & 1,5 & 7,6 & 1,5 & 6,1 & 100,0 \\
\hline $45-49$ & 77,2 & 8,8 & 7,0 & 1,8 & - & 5,3 & 100,0 \\
\hline Total & 42,9 & 35,3 & 5,2 & 4,8 & 1,6 & 10,2 & 100,0 \\
\hline
\end{tabular}

Figura 3

Distribuição das mulheres segundo motivo da escolha do MAC que usam atualmente.

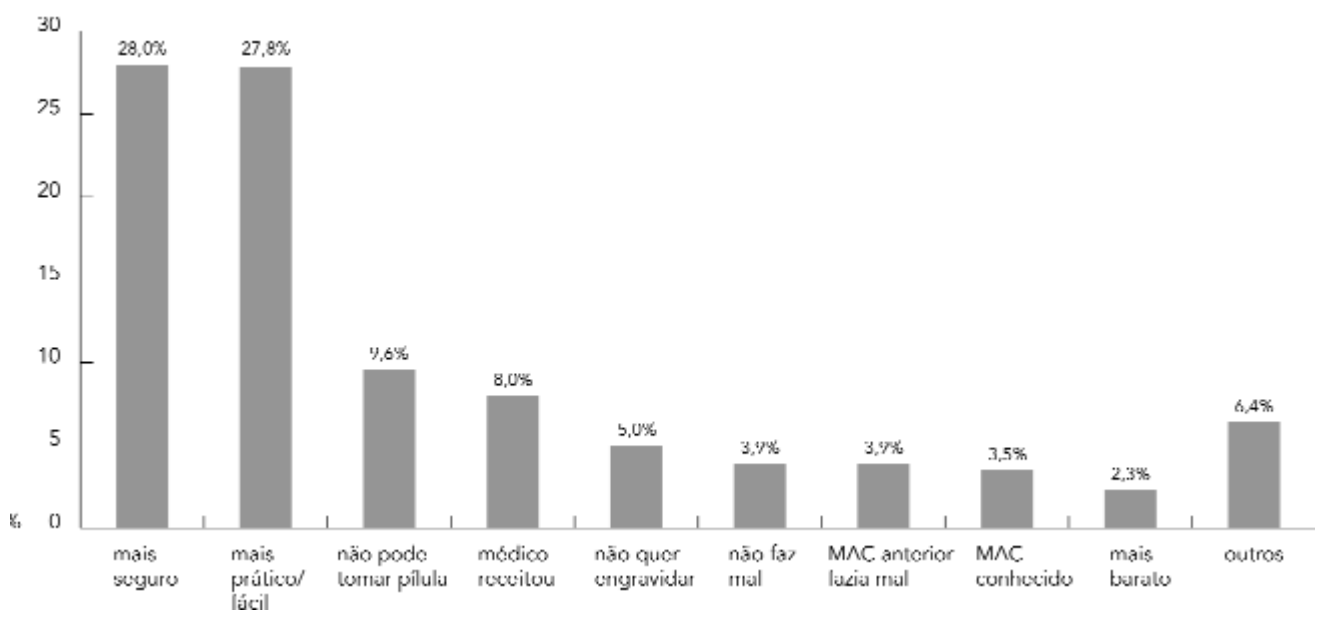




\section{Considerações Finais}

Buscou-se apontar, neste trabalho, as questões relativas ao conhecimento e uso de métodos anticoncepcionais entre mulheres residentes na Região Sul do Município de São Paulo, no ano de 1992.

Inicialmente foi observado que, nas questões respeitantes ao conhecimento de métodos contraceptivos, $86,4 \%$ das mulheres referiram conhecer pelo menos um dos métodos disponíveis no mercado, enquanto $13,6 \%$ nunca ouviram falar em métodos. Esse grupo é composto, em sua grande maioria, por adolescentes com idade inferior aos 14 anos. Mas, apesar do alto índice de conhecimento de MAC, constatou-se baixo uso do preservativo, que ficou em torno de 5,2\% apesar do advento da AIDS, e alta freqüência de esterilizações femininas $(42,9 \%)$, que supera o uso da pílula $(35,3 \%)$. Na prática, a contracepção fica circunscrita à esterilização feminina e à pílula.

Comentando a situação da mulher brasileira em relação à contracepção, Marcolino (1994:53) menciona: “À primeira vista, podería- mos dizer que a mulher brasileira está muito bem servida quanto à oportunidade da contracepção, mas uma observação mais cuidadosa dos dados mostra-nos que a situação não é tão favorável assim, pois elas se concentram em dois métodos: a pílula e a esterilização. O peso desses dois métodos nas alternativas contraceptivas denota a precariedade de opção para a mulher brasileira".

Os programas de atenção à saúde da mulher não estão cumprindo a assistência que as mulheres necessitam, principalmente a orientação e o acompanhamento das questões relacionadas às práticas contraceptivas e à escolha do método a ser utilizado. Além disso, não incentivam a participação masculina no processo de regulação da fecundidade.

Os resultados do presente estudo reforçam a convicção de que a sociedade e seus dirigentes devem efetivamente voltar seus esforços para garantir a consolidação dos programas de atenção à saúde da mulher, enfatizando a informação, a orientação e o acesso à anticoncepção, tomando em conta os princípios dos direitos reprodutivos.

\section{Agradecimentos}

Os autores agradecem o apoio financeiro do Conselho Nacional de Desenvolvimento Científico e Tecnológico - CNPq e da Fundação de Amparo à Pesquisa do Estado de São Paulo - FAPESP.

\section{Referências}

BEMFAM (Sociedade Civil Bem-Estar Familiar no Brasil), 1997. Brasil-Pesquisa Nacional sobre Demografia e Saúde - 1996. Rio de Janeiro: BEMFAM.

BERQUÓ, E., 1993. Brasil - Um Caso Exemplar: Anticoncepção e Partos Cirúrgicos. Trabalho apresentado no Seminário "A Situação da Mulher e o Desenvolvimento”. Campinas: Núcleo de Estudos Populacionais - NEPO, Universidade Estadual de Campinas - UNICAMP. (mimeo.)

BERQuÓ, E.; MORELL, M. G. G. P.; SILVA, R. S. \& MARQUES, R. M., 1985. São Paulo e sua fecundidade: Um estudo das variáveis intermediárias e dos diferenciais socioeconômicos da fecundidade. In: Série São Paulo 80, vol. 1, pp. 18-30, São Paulo: Fundação Sistema Estadual de Análise de Dados - SEADE.

IBGE (Fundação Instituto Brasileiro de Geografia e Estatística), 1991. Sinopse Preliminar do Censo Demográfico. São Paulo: IBGE. 
MARCOLINO, C., 1994. Trajetória da Mulher em Direção à Esterilização Cirúrgica Feminina: Um Estudo Fenomenológico. Dissertação de Mestrado, São Paulo: Faculdade de Saúde Pública, Universidade de São Paulo.

MORELL, M. G. G. P., 1994. Anticoncepção em São Paulo em 1986: Prevalência e características. Informe Demográfico, 25:199-333.

OSIS, M. J. M. D., 1994. Atenção Integral à Saúde da Mulher, o Conceito e o Programa: História de uma Intervenção. Dissertação de Mestrado, Campinas: Instituto de Filosofia e Ciências Humanas, Universidade Estadual de Campinas.

PIROTTA, K. M., 1998. A Mulher e a Esterilização: Do Mito da Emancipação ao Desvelamento da Subalternidade. Dissertação de Mestrado, São Paulo: Faculdade de Saúde Pública, Universidade de São Paulo.

SCHOR, N., 1995. Adolescência e Anticoncepção: Conhecimento e Uso. Tese de Livre Docência, São Paulo: Faculdade de Saúde Pública, Universidade de São Paulo.

SCHOR, N. \& MORELL, M. G. G. P., 1994. Saúde Reprodutiva: Uma Tentativa de Caracterização das Condições de Reprodução em São Paulo. Relatório de pesquisa. São Paulo: Conselho Nacional de Desenvolvimento Científico e Tecnológico - CNPq. (mimeo.)
SIQUEIRA, A. A. F.; TANAKA, A. C. d'A.; MORON, A. F; ALVARENGA, A. T.; UNGLERT, C. V. S.; SCHOR, N. \& SANTOS, J. L. F., 1991. Estudo da Mortalidade Materna na Região Sul do Município de São Paulo, Brasil: Análise Preliminar. Série Investigação em Saúde da Mulher, da Criança e Adolescência, 1. São Paulo: Faculdade de Saúde Pública, Universidade de São Paulo.

SIQUEIRA, A. A. F.; TANAKA, A. C. d'A.; MORON, A. F.; ALVARENGA, A. T.; UNGLERT, C. V. S.; SCHOR, N.; REIS, A. O. A. \& SANTOS, J. L. F., 1993. Morbidade e Mortalidade Maternas, Qualidade da Assistência e Estrutura Social: Estudo da Região Sul do Município de São Paulo - Brasil. Parte II. Morbidade Materna. Relatório de Pesquisa. São Paulo: Conselho Nacional de Desenvolvimento Científico e Tecnológico - CNPq. (mimeo.)

UNGLERT, C. V. S., 1986. Contribuição para Estudo da Localização de Serviços de Saúde: Uma Abordagem Geográfica em Saúde Pública. Tese de Doutorado, São Paulo: Faculdade de Saúde Pública, Universidade de São Paulo.

UNGLERT, C. V. S.; SCHOR, N.; PERES, F.; WEILLER, R. M.; JUNQUEIRA, C. B.; SIQUEIRA, A. A. F. \& ARENO, F. B., 1990. Enfoque epidemiológico de la mortalidad em menores de un año Región Sur del município de San Pablo, Brasil. Medicina e Sociedade, 13:16-23. 